\title{
ARE WE PREPARED TO USE BAG-MASK-VENTILATION DURING CONTINUOUS CHEST COMPRESSION?
}

\author{
Michael Czekjalo ${ }^{1}$ \\ 'Department of Surgery, Hunter Holmes McGuire VA Medical Center, Richmond, USA, United States
}

Disaster Emerg Med J 2018; 3(4): 148-149

Sir,

Ventilation of the patient during cardiopulmonary resuscitation is an important element of resuscitation procedures. Optimal oxygenation of the patient increases the chances of a return of spontaneous circulation without significant neurological defects resulting from brain tissue hypoxia $[1,2]$. Jabre et al. indicated that among patients with out-of-hospital cardiorespiratory arrest, the use of bag-mask ventilation (BMV) compared with endotracheal intubation (ETI) failed to demonstrate non-inferiority or inferiority for survival with favorable 28-day neurological function [3].

Ventilation with BMV is the first ventilation method undertaken by medical personnel in the conditions of medical rescue teams as well as in -hospital cardiac arrest settings. However, poor ventilation of the patient with BMV may result in air insufflation to the stomach, increasing gastric pressure and diaphragm shift, which reduces the effectiveness of chest compressions and even increases the risk for stomach rupture $[4,5]$. It is also worth emphasizing that excessively increased gastric pressure may lead to regurgitation of gastric contents and its aspirations. Nevertheless, ventilation with BMV may seem like an easy procedure, but numerous studies indicate that emergency personnel often use the laryngeal mask to ventilate incorrectly $[6,7]$. Significantly better ventilation effects are achieved with double C-E or V-E grip [8]. However, this requires the involvement of two persons to ventilate the patient, which is impossible to achieve under EMS conditions.

The aim of the study was to evaluate the effectiveness of bag-mask ventilation performed by nurses under conditions of simulated adult cardiopulmonary resuscitation.

The study was carried out in 64 nurses taking part in certified pieces of training in Basic Life Support accredited by the American Heart Association. Nurses specializing in emergency medicine or anesthesiology were excluded from the study. Voluntary written informed consent was obtained from each participant. Before the study, participants were presented with a correct BMV with the use of two techniques: (A) standard C-E technique, (B) double technique C-E. Ventilation took place during simulated 2-minute cardiopulmonary resuscitation. After ventilation with one method, the participants had a 10-minute break and then performed ventilation with another technique. Both the order of participants and study methods were randomized. The chest compressions were performed using the LUCAS3 chest compression system set to 30 chest compressions: 2 rescue breaths. Ventilation parameters were analyzed. At the end of the study, the participants assessed their preferences for ventilation using the tested techniques.

The study involved 64 nurses with an average age of $43.5 \pm 12$ years and an average work experience of $23 \pm 10.5$ years. In the case of the C-E technique, only $35.9 \%$ of all ventilation attempts ended with a correct rescue breathing, and in the case of the double $C$-E technique $-93.8 \%(P<0.001)$. Average ventilation volume in the case of effective ventilation obtained in both single and dual C-E techniques was differentiated and amounted to $760 \mathrm{~mL} \pm 120$ vs. $880 \mathrm{~mL} \pm 190(P=0.011)$, respectively. $90.6 \%$ of the study participants chose 
the double C-E technique as the preferred ventilation technique.

In conclusion, BMV is an important element of the rescue procedure. During simulated resuscitation, the dual C-E grip technique during BMV increases the effectiveness of patient ventilation. The adult tidal volume should be 6-7 mL per kilogram of body weight. It is necessary to implement systematic training in BMV, as the ventilation volumes obtained by nurses are much higher than recommended for this group of patients, which may affect the previously mentioned complications, including gastric ventilation and increased risk of regurgitation. An alternative to BMV may be supraglottic airway devices.

\section{REFERENCES:}

1. Smereka J, Iskrzycki L, Makomaska-Szaroszyk E, et al. The effect of chest compression frequency on the quality of resuscitation by lifeguards. A prospective randomized crossover multicenter simulation trial. Cardiol J. 2018 [Epub ahead of print], doi: 10.5603/ CJ.a2018.0121, indexed in Pubmed: 30338845.

2. Klosiewicz T, Sip M, Zalewski R. Alternative of endotracheal intubation for paramedics to provide direct laryngoscopy. The randomized man- ikin trial. Disaster Emerg Med J. 2017; 2(3): 142-144, doi: 10.5603/ DEMJ.a2017.0032.

3. Jabre P, Penaloza A, Pinero D, et al. Effect of Bag-Mask Ventilation vs Endotracheal Intubation During Cardiopulmonary Resuscitation on Neurological Outcome After Out-of-Hospital Cardiorespiratory Arrest: A Randomized Clinical Trial. JAMA. 2018; 319(8): 779-787, doi: 10.1001/jama.2018.0156, indexed in Pubmed: 29486039.

4. Frass M, Robak 0, Smereka J, et al. Securing the airway patency by firefighters with the use of CombiTube. A pilot data. Disaster Emerg Med J. 2018; 3(2): 46-50, doi: 10.5603/demj.2018.0011.

5. Bednarz S, Filipovic M, Schoch 0 , et al. Gastric rupture after bag-mask-ventilation. Respir Med Case Rep. 2015; 16: 1-2, doi: 10.1016/j.rmcr.2015.05.014, indexed in Pubmed: 26744639.

6. Sip M, Dabrowska A, Prucnal K, et al. Supraglottic devices - future or everyday life? Disaster Emerg Med J. 2017; 2(2): 74-83, doi: 10.5603/DEMJ.2017.0015.

7. Majer J, Jaguszewski MJ, Frass M, et al. Does the use of cardiopulmonary resuscitation feedback devices improve the quality of chest compressions performed by doctors? A prospective, randomized, cross-over simulation study. Cardiol J. 2018 [Epub ahead of print], doi: 10.5603/CJ.a2018.0091, indexed in Pubmed: 30155865.

8. Soleimanpour M, Rahmani F, Morteza Bagi HR, et al. Comparison of Three Techniques on Facility of Bag-Mask Ventilation: Thenar Eminence, E-0 and E-C. Anesth Pain Med. 2018; 8(4): e74226, doi: 10.5812/aapm.74226, indexed in Pubmed: 30250820. 